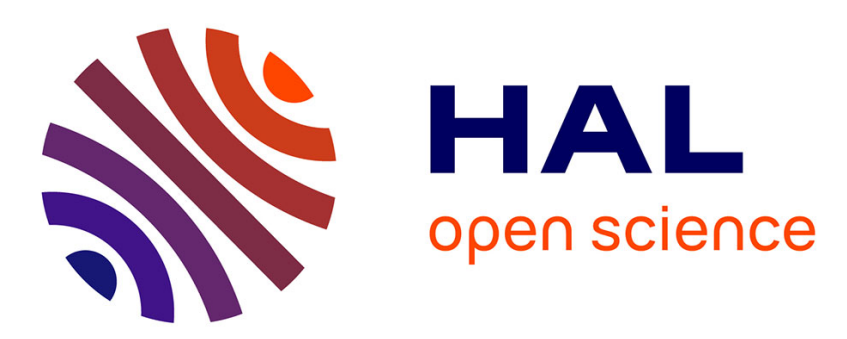

\title{
Consensus under switching spanning-tree topologies and persistently exciting interconnections
}

Nohemi Alvarez-Jarquin, Antonio Loria, José Luis Avila

\section{To cite this version:}

Nohemi Alvarez-Jarquin, Antonio Loria, José Luis Avila. Consensus under switching spanningtree topologies and persistently exciting interconnections. Proc. IEEE American Control Conference (ACC), Jun 2018, Milwaukee, United States. pp.4578-4583, 10.23919/acc.2018.8431206 . hal01753415

\section{HAL Id: hal-01753415 \\ https://hal.science/hal-01753415}

Submitted on 29 Mar 2018

HAL is a multi-disciplinary open access archive for the deposit and dissemination of scientific research documents, whether they are published or not. The documents may come from teaching and research institutions in France or abroad, or from public or private research centers.
L'archive ouverte pluridisciplinaire HAL, est destinée au dépôt et à la diffusion de documents scientifiques de niveau recherche, publiés ou non, émanant des établissements d'enseignement et de recherche français ou étrangers, des laboratoires publics ou privés. 


\title{
Consensus under switching spanning-tree topologies and persistently exciting interconnections
}

\author{
Nohemi Alvarez-Jarquin ${ }^{1} \quad$ Antonio Loría ${ }^{2} \quad$ José Luis Avila $^{1}$
}

\begin{abstract}
We study the consensus problem for networks with changing communication topology and with time-dependent communication links. That is, the network changes in two dimensions: "geographical" and "temporal". We establish that consensus is reached provided that there always exists a spanning tree for a minimal dwell-time and the interconnection gains are persistently exciting. Our main result covers the particular case, studied in the literature, of one fixed-topology with time-varying interconnections but also that of changing topologies with reliable interconnections during a dwell-time. Another originality of our work lies in the method of proof, based on stability theory of time-varying and switched systems. Simulations on an academic example are provided to illustrate our theoretical results.
\end{abstract}

\section{INTRODUCTION}

In spite of the considerable bulk of literature on consensus consensus analysis, such problem for systems with taime-varying changing topologies and time-varying interconnections, has been little studied; some recent works include [2], [8], [3], [9]. This problem, however, is of great interest for researchers of several disciplines due to the multiple applications related to networked multiagent systems: satellite formation flying [1], [14], coupled oscillators, formation tracking control for mobile robots [4], coupled air traffic control [15] just to mention a few. These applications justify the design of appropriate consensus protocols to drive all dynamic agents to a common value. The consensus problem consists in establishing conditions under which the differences between any two motions among a group of dynamic systems, converge to zero asymptotically.

In [12] the authors present multiple agents in the presence of limited and unreliable information exchange with changing communication topologies; the analysis relies on the graph theory and consensus may be established if the union of the directed interaction graphs have a spanning tree. In [11] directed networks with switching topology are treated as a hybrid system. A common Lyapunov function allows to show convergence analysis of an agreement protocol for this system.

The authors of [5] study the consensus problem in undirected networks of dynamic agents with fixed and switching topologies. Using Lyapunov theory; it is showed that

A. Loría is with CNRS. Address: LSS-SUPELEC, 91192 Gif-sur-Yvette, France. E-mail: antonio.loria@lss.supelec.fr. N. Alvarez-Jarquin and J. L. Avila are with Laboratorio Nacional de Geointeligencia, CentroGeo Aguascalientes, 20313 Circuito Tecnopolo Sur, Aguascalientes, México. Email: nalvarez@centrogeo.edu.mx, javila@centrogeo.edu.mx. The work of A. Loría was sponsored by the Dept. STIC of Univ. Paris Saclay, France. all the nodes in the network achieve consensus asymptotically for appropriate communication delays, if the network topology graph is connected. In [16] the authors address the consensus problem for discrete-time multiagent systems with changing communications topologies and bounded time-varying communication delays.

In this paper, we consider the consensus problem for networks of dynamic systems interconnected in a directed graph through time-varying links. In contrast to the related literature, see for instance [7], [17], we assume that the network's graph is time-varying with respect to two time-scales. Firstly, we assume that the interconnection topology changes that is, the agent $A$ may communicate with $B$ over a period of time and with $C$ over a (dwell-)time interval. Secondly, in clear contrast with the literature, we assume that during a dwell-time in which the topology is fixed, the communication links are not reliable that is, they vary with time and, in particular, they may have random failures. The necessary and sufficient condition is that each interconnection gain is, separately, persistently exciting. Persistently exciting covers in particular random signals of positive mean (offset). This is also in contrast to conditions based on excitation of an "averaged" graph Laplacian -cf. [7]. Thus, the problem we analyze covers both the case of fixed topology with time-varying interconnections and that of switching topologies with reliable interconnections.

In the following section we present our main results. For clarity of exposition, we present in the first place, an auxiliary result on consensus under a fixed spanning tree topology with time-varying reliable interconnections. Then, we show that the switched topology problem may be approached using stability theory for switched linear time-varying systems. In Section III we present an example of three agents whose interconnection topology changes among six possible configurations. Concluding remarks are provided in Section IV.

\section{MAIN RESULTS}

\section{A. Problem statement}

Consider $N$ dynamic agents

$$
\Psi_{\lambda}: \dot{x}_{\lambda}=u_{\lambda}, \quad \lambda=1,2, \cdots, N
$$

where $u_{\lambda}$ represents a protocol of interconnection. The most common continuous consensus protocol under an all-to-all communication assumption, is given by -see [11], [16],

$$
u_{\lambda}(t, x)=-\sum_{\kappa=1}^{N} a_{\lambda \kappa}(t)\left[x_{\lambda}(t)-x_{\kappa}(t)\right],
$$


where $a_{\lambda \kappa}$ is the $(\lambda, \kappa)$ entry of the adjacency matrix and $x_{\lambda}$ is the information state of the $\lambda$-th agent.

The system (1), (2) reaches consensus if for every initial condition all the states reach a common value as $t$ tends to infinity. The consensus problem has been thoroughly studied both for the case of constant and time-varying interconnections, mostly under the assumption of an allto-all communication topology. It also is well known, from graph theory, that it is necessary and sufficient to reach consensus that there exists a spanning tree. In the case that the interconnections are time-varying, a similar result was established in [10] based on the assumption that, roughly speaking, there exists an average spanning tree.

In this paper, we analyze consensus under time-varying topologies; as opposed to the more traditional graphtheory based analysis [13], we adopt a stability theory approach.

\section{B. The network model}

With little loss of generality, let us consider the following consensus protocol

$u_{\lambda}= \begin{cases}-a_{\lambda \lambda+1}(t)\left[x_{\lambda}(t)-x_{\lambda+1}(t)\right], & \forall \lambda \in[1, N-1], \\ 0, & \lambda=N,\end{cases}$

where $a_{\lambda \lambda+1} \geq 0$ and it is strictly positive whenever information flows from the $(\lambda+1)$ th node to the $\lambda$ th node. This protocol leads to a spanning-tree configuration topology; the closed-loop equations are

$$
\begin{cases}\dot{x}_{1} & =-a_{12}(t)\left[x_{1}-x_{2}\right] \\ & \vdots \\ \dot{x}_{\lambda} & =-a_{\lambda, \lambda+1}(t)\left[x_{\lambda}-x_{\lambda+1}\right] \\ & \vdots \\ \dot{x}_{N-1} & =-a_{N-1 N}(t)\left[x_{N-1}-x_{N}\right] \\ \dot{x}_{N} & =0\end{cases}
$$

In a leader-follower configuration, the $N$ th node may be considered as a "swarm master" with its own dynamics. For simplicity, here we consider it to be static.

It is clear that there are many other possible spanningtree configurations; the one showed above is considered conventionally. Actually, there exist a total number of $N$ ! spanning-tree configurations; for instance, for a group of three agents there exist six possible spaning-tree configuration topologies which determine six different sequences

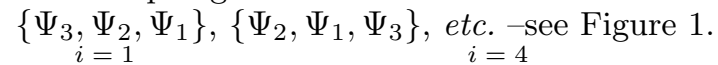

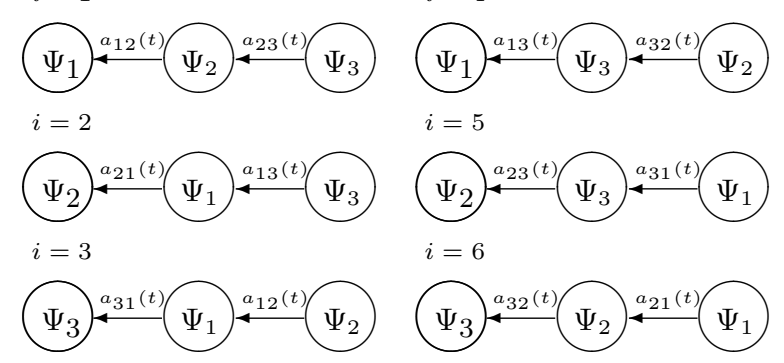

Fig. 1. Example of 3 agents, where by changing their positions, we obtained six possible topologies.
Thus, to determine the $N$ ! possible spanning-tree communication topologies, among $N$ agents, we introduce the following notation. For each $k \leq N$ we define a function $\pi_{k}$ which takes integer values in $\{1, \ldots N\}$. We also introduce the sequence of agents $\left\{\Psi_{\pi_{k}}\right\}_{k=1}^{N}$ with the following properties: 1 ) every agent $\Psi_{\lambda}$ is in the sequence; 2) no repetitions of agents in the sequence is allowed 3) the root agent is labeled $\Psi_{\pi_{N}}$ and it communicates with the agent $\Psi_{\pi_{N-1}}$, the latter is parent of $\Psi_{\pi_{N-2}}$ and so on down to the leaf agent $\Psi_{\pi_{1}}$. That is, the information flows with interconnection gain $a_{\pi_{k} \pi_{k+1}}(t) \geq 0$, from the agent $\Psi_{\pi_{k+1}}$ to the agent $\Psi_{\pi_{k}}$. The subindex $k$ represents the position of the agent $\Psi_{\pi_{k}}$ in the sequence. Note that any sequence $\left\{\Psi_{\pi_{1}}, \Psi_{\pi_{2}}, \ldots, \Psi_{\pi_{N-1}}, \Psi_{\pi_{N}}\right\}$ of the agents may be represented as a spanning-tree topology which is depicted in Figure 2.

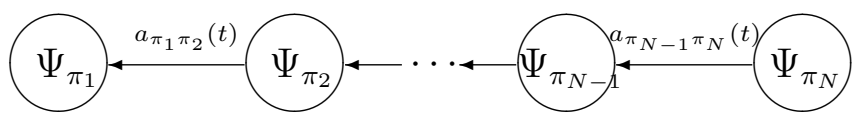

Fig. 2. A spanning-tree topology with time dependent communication links between $\Psi_{\pi_{k}}$ and $\Psi_{\pi_{k+1}}$.

Thus, in general, each possible fixed topology labeled $i \in 1, \ldots, N$ ! is generated by a protocol of the form (3) which we write as

$u_{\pi_{k}}^{i}=\left\{\begin{array}{cl}-a_{\pi_{k} \pi_{k+1}}^{i}(t)\left[x_{\pi_{k}}-x_{\pi_{k+1}}\right], & k \in\{1, \ldots, N-1\}, \\ 0, & k=N,\end{array}\right.$

where $k$ denotes the position of the agent $\Psi_{\lambda}$ in the sequence $\left\{\Psi_{\pi_{k}}\right\}_{k=1}^{N}$ and $\pi_{k}$ represents which agent $\Psi_{\lambda}$ is in the position $k$, this is, $\pi_{k}=\lambda$. Under (5), the system (1) takes the form

$$
\dot{x}_{d i}=-L_{i}(t) x_{d i}, \quad i \in\{1, \ldots, N !\},
$$

where to each topology $i \leq N$ ! corresponds a state vector

$$
x_{d i}=\left[x_{\pi_{1}}, x_{\pi_{2}}, \ldots, x_{\pi_{N}}\right]^{\top}
$$

which contains the states of all interconnected agents in a distinct order, depending on the topology. For instance, referring to Figure 1 , for $i=1$ we have $x_{d 1}=\left[x_{1}, x_{2}, x_{3}\right]^{\top}$ while $x_{d 4}=\left[x_{1}, x_{3}, x_{2}\right]^{\top}$ while for $i=4$.

Accordingly, to each topology we associate a distinct Laplacian matrix $L_{i}(t)$ which is given by

$$
\begin{gathered}
L_{i}(t):= \\
{\left[\begin{array}{ccccc}
a_{\pi_{1} \pi_{2}}^{i}(t) & -a_{\pi_{1} \pi_{2}}^{i}(t) & 0 & 0 & 0 \\
0 & a_{\pi_{2} \pi_{3}}^{i}(t) & -a_{\pi_{2} \pi_{3}}^{i}(t) & 0 & 0 \\
\vdots & \vdots & \ddots & \ddots & \vdots \\
0 & 0 & \ldots & a_{\pi_{k-1} \pi_{k}}^{i}(t) & -a_{\pi_{k-1} \pi_{k}}^{i}(t) \\
0 & 0 & \ldots & \cdots & 0
\end{array}\right]}
\end{gathered}
$$

Since any of the $N$ ! configurations is a spanning tree, which is a necessary and sufficient condition for consensus, all configurations may be considered equivalent in some sense, to the first topology, i.e., with $i=1$. As a convention, for the purpose of analysis we denote the state of the latter by $x=\left[x_{1}, x_{2}, \ldots, x_{N}\right]^{\top}$ and refer to it as an ordered topology. See Figure 3. 


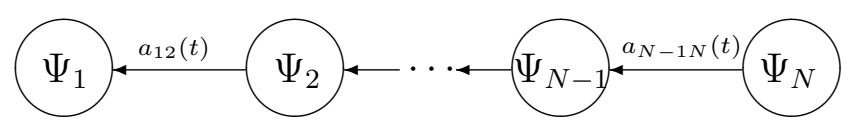

Fig. 3. A spanning-tree topology with time dependent communication links between $\Psi_{\pi_{k}}$ and $\Psi_{\pi_{k+1}}$.

It is clear (at least intuitively) that consensus of all systems (6) is equivalent to that of $\dot{x}=L_{1}(t) x$, where

$$
\begin{aligned}
& L_{1}(t):= \\
& {\left[\begin{array}{ccccc}
a_{12}(t) & -a_{12}(t) & 0 & 0 & 0 \\
0 & a_{23}(t) & -a_{23}(t) & 0 & 0 \\
\vdots & \vdots & \ddots & \ddots & \vdots \\
0 & 0 & \ldots & a_{N-1 N}(t) & -a_{N-1 N}(t) \\
0 & 0 & \ldots & 0 & 0
\end{array}\right] .}
\end{aligned}
$$

More precisely, the linear transformation from a "disordered" vector $x_{d i}$ to the ordered vector $x$ is defined via a permutation matrix $P_{i}$ that is,

$$
x_{d i}=P_{i} x
$$

with $P_{i} \in \mathbb{R}^{n \times n}$ defined as

$$
P_{i}=\left[\begin{array}{c}
E_{\pi_{1}} \\
E_{\pi_{2}} \\
\vdots \\
E_{\pi_{N}}
\end{array}\right], \quad i \in\{1, \ldots, N !\}
$$

and the rows

$$
E_{\pi_{k}}=[0,0, \ldots, \underbrace{1}_{\pi_{k} \text { th position }} \ldots, 0] .
$$

The permutation matrix $P_{i}$ is a nonsingular matrix with $P_{i}^{-1}=P_{i}^{\top}[6]$. For instance, relative to Figure 1 we have $x_{d 2}=\left[x_{2}, x_{1}, x_{3}\right]^{\top}$ and

$$
P_{2}=\left[\begin{array}{lll}
0 & 1 & 0 \\
1 & 0 & 0 \\
0 & 0 & 1
\end{array}\right]
$$

In order to study the consensus problem for (6) for any $i$ it is both sufficient and necessary to study that of any configuration topology. Moreover, we may do so by studying the error dynamics corresponding to the differences between any pair of states.

\section{Fixed topology}

For clarity of exposition we start with a preliminary result which applies to the case of a fixed but arbitrary topology $-c f$. [13, Theorem 2.33]. In view of the previous discussion, without loss of generality, we focus on the study of the ordered topology depicted in Figure 3. Consensus may be established using an argument on stability of cascaded systems. To see this, let $z_{1}$ denote the vector of ordered errors corresponding to this first topology that is,

$$
z_{1 \lambda}:=x_{\lambda}-x_{\lambda+1}, \quad \forall \lambda \in\{1, \ldots, N-1\} .
$$

Then, the systems in (6) with $i=1$ reach consensus if and only if the origin of

$$
\begin{aligned}
\dot{z}_{11} & =-a_{12}(t) z_{11}+a_{23}(t) z_{12} \\
\dot{z}_{12} & =-a_{23}(t) z_{12}+a_{34}(t) z_{13} \\
& \vdots \\
\dot{z}_{1 N-1} & =-a_{N-1 N}(t) z_{1 N-1}
\end{aligned}
$$

is (globally) uniformly asymptotically stable.

In a fixed topology we have $a_{\lambda, \lambda+1}(t)>0$ for all $t \geq 0$ that is, the $\lambda$ th node in the sequence always receives information from its parent labeled $\lambda+1$, albeit with varying intensity. The origin of the decoupled bottom equation, which corresponds to the dynamics of the root node, is uniformly exponentially stable if $a_{N-1 N}(t)>0$ for all $t$. Each of the subsystems in (11) from the bottom to the top is input to state stable. Uniform exponential stability of the origin $\{z=0\}$ follows provided that $a_{\lambda \lambda+1}$ is bounded.

In compact form, the consensus dynamics becomes

$$
\dot{z}_{1}=A_{1}(t) z_{1}, \quad z_{1}=\left[z_{11} \cdots z_{1 N-1}\right]^{\top}
$$

where the matrix $A_{1}(t) \in \mathbb{R}^{N-1 \times N-1}$ is defined as

$A_{1}(t)=$

$\left[\begin{array}{ccccc}-a_{12}(t) & a_{23}(t) & 0 & \cdots & 0 \\ 0 & -a_{23}(t) & a_{34}(t) & 0 & \vdots \\ \vdots & \vdots & \ddots & \ddots & \vdots \\ 0 & 0 & 0 & -a_{N-2 N-1}(t) & a_{N-1 N}(t) \\ 0 & 0 & 0 & \cdots & -a_{N-1 N}(t)\end{array}\right]$.

Lemma 1: Let

$\dot{\Phi}\left(t ; t_{\circ}\right)=A_{1}(t) \Phi\left(t ; t_{\circ}\right), \quad \Phi\left(t_{\circ} ; t_{\circ}\right)=I_{N-1}, \quad \forall t \geq t_{\circ}>0$.

Assume that, for every $i \in\{1, \ldots, N-1\}, a_{i i+1}$ is a bounded persistently exciting signal that is, there exist $T_{i}$ and $\mu_{i}>0$ such that

$$
\int_{t}^{t+T_{i}} a_{i i+1}(s) d s \geq \mu_{i} \quad \forall t \geq 0 .
$$

Then, there exist $\bar{\alpha}>0, \alpha>0$ such that

$$
\left\|\Phi\left(t ; t_{\circ}\right)\right\| \leq \bar{\alpha} e^{-\alpha\left(t-t_{\circ}\right)}, \quad \forall t \geq t_{\circ} \geq 0 .
$$

Proof: Note that the solution of the differential equation (14) is given by $\Phi\left(t ; t_{\circ}\right)=\left[\phi_{i j}\left(t ; t_{\circ}\right)\right]$, where

$\phi_{i j}\left(t ; t_{\circ}\right)=\left\{\begin{array}{cc}e^{0,} & i>j, \\ e^{-\int_{t_{\circ}}^{t} a_{i i+1}(s) d s}, & i=j, \\ \int_{t_{\circ}}^{t} \phi_{i i}(t ; s) a_{i+1, i+2}(s) \phi_{i+1, j}\left(s ; t_{\circ}\right) d s, & i<j .\end{array}\right.$

We show that every element of $\Phi\left(t ; t_{\circ}\right)$ is bounded by an exponentially-decreasing function. Using the Schwartz 
inequality for the third integral and (15) for the second we obtain

$$
\begin{array}{rrr}
\left|\phi_{i i}\left(t ; t_{\circ}\right)\right| & \leq \bar{k}_{i} e^{-k_{i}(t-s)}, & i=j ; \\
\left|\phi_{i j}\left(t ; t_{\circ}\right)\right| & \leq \int_{t_{\circ}}^{t}\left|\phi_{i i}(t ; s)\right|\left|a_{i+1, i+2}(s)\right|\left|\phi_{i+1, j}\left(s ; t_{\circ}\right)\right| d s \\
& & i<j .
\end{array}
$$

For each $j=i+1$ such that $i<N-1$ the third integral in (17) depends on $\phi_{i i}$ and $\phi_{i+1 i+1}$ which are bounded by $\bar{k}_{i} e^{-k_{i}(t-s)}$ and $\bar{k}_{i+1} e^{-k_{i+1}(t-s)}$, respectively. Consequently,

$\left|\phi_{i j}\left(t ; t_{\circ}\right)\right| \leq \bar{k}_{i} \bar{k}_{j}\left|a_{i+1, i+2}\right|_{\infty}\left[\frac{1}{\left|k_{i}-k_{j}\right|} e^{-\min \left\{k_{j}, k_{i}\right\}\left(t-t_{\circ}\right)}\right]$

where by assumption, $\left|a_{i+1, i+2}\right|_{\infty}$ is bounded. Thus, all elements of $\Phi_{i j}\left(t ; t_{\circ}\right)$ are bounded in norm by a decaying exponential.

\section{Time-varying topology}

In this section we study the more general case, in which not only the interconnection gains are time-varying, as in the previous section and [13], but the topology may be randomly chosen as long as there always is a spanning tree which lasts for at least a dwell-time.

For the purpose of analysis we aim at identifying, with each possible topology, a linear time-varying system of the form (12) with a stable origin and to establish stability of the switched system. To that end, let $i$ determine one among the $N$ ! topologies schematically represented by a graph as showed in Figure 2. Let $x_{\lambda}$ denote the state of system $\Psi_{\lambda}$ then, for the $i$ th topology, we define the error

$$
\begin{aligned}
z_{i} & =\left[z_{i 1} \cdots z_{i N-1}\right]^{\top}, \\
z_{i k} & =x_{\pi_{k}}-x_{\pi_{k+1}}, \quad k \in\{1, \cdots, N-1\},
\end{aligned}
$$

where $k$ denotes the graphical position of the agent $\Psi_{\lambda}$ in the sequence $\left\{\Psi_{\pi_{k}}\right\}_{k=1}^{N}$ and $\pi_{k}$ represents which agent $\Psi_{\lambda}$ is in the position $j$, this is, $\pi_{k}=\lambda$.

Example 1: Consider two possible topologies among those showed in Figure 1 represented in more detail in Figure 4 (for $i=1$ ) and Figure 5 (for $i=4$ ). Then, we have

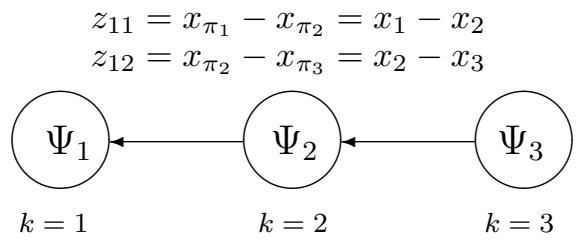

Fig. 4. A topology with 3 agents where $\pi_{1}=1, \pi_{2}=2$ and $\pi_{3}=3$.

whereas in the second case, when $i=4$,

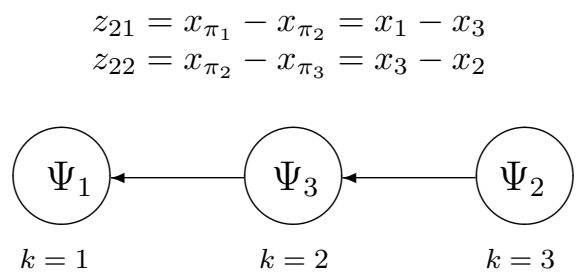

Fig. 5. The second topology with 3 agents where $\pi_{1}=1, \pi_{2}=3$ and $\pi_{3}=2$.
That is, for each topology $i$ the dynamics of the interconnected agents is governed by the equation

$$
\dot{z}_{i}=A_{i}(t) z_{i}
$$

where

$A_{i}(t):=$

$$
\left[\begin{array}{cccc}
-a_{\pi_{1} \pi_{2}}^{i}(t) & a_{\pi_{2} \pi_{3}}^{i}(t) & 0 & 0 \\
\vdots & \ddots & \ddots & \vdots \\
0 & 0 & -a_{\pi_{N-2} \pi_{N-1}}^{i}(t) & a_{\pi_{N-1} \pi_{N}}^{i}(t) \\
0 & 0 & \cdots & -a_{\pi_{N-1} \pi_{N}}^{i}(t)
\end{array}\right]
$$

According to Lemma 1 the origin $\left\{z_{i}=0\right\}$ is uniformly globally exponentially stable provided that $a_{\pi_{k} \pi_{k+1}}(t)$ is strictly positive for all $t$. It is clear that consensus follows if the origin $\left\{z_{i}=0\right\}$ for any of the systems (21) (with $i$ fixed for all $t$ ) is uniformly exponentially stable. Actually, there exist $\alpha_{i}$ and $\bar{\alpha}_{i}$ such that

$$
\left|z_{i}(t)\right| \leq \bar{\alpha}_{i} e^{-\alpha_{i} t}, \quad \forall t \geq 0 .
$$

Observing that all the systems (21) are equivalent up to a linear transformation, our main result establishes consensus under the assumption that topology changes, provided that there exists a minimal dwell-time. Indeed, the coordinates $z_{i}$ are related to $z_{1}$ by the transformation

$$
z_{i}=W_{i} z_{1}
$$

where $W_{i}:=T P_{i} T^{-1}, P_{i}$ is defined in (10), $T \in \mathbb{R}^{N-1 \times N}$ is given by

$$
T=\left[\begin{array}{cccccc}
1 & -1 & 0 & \cdots & 0 & 0 \\
0 & 1 & -1 & \cdots & 0 & 0 \\
\vdots & & \ddots & \ddots & & \vdots \\
0 & 0 & 0 & 1 & -1 & 0 \\
0 & 0 & 0 & \cdots & 1 & -1
\end{array}\right]
$$

and $T^{-1} \in \mathbb{R}^{N \times N-1}$ denotes a right inverse of $T$. Note that the matrix $W_{i} \in \mathbb{R}^{N-1 \times N-1}$ is invertible for each $i \leq$ $N$ ! since each of its rows consists in a linear combination of two different rows of $T^{-1}$, which contains $N-1$ linearlyindependent rows. Actually, using (24) in (21) we obtain

$$
\dot{z}_{1}=\bar{A}_{i}(t) z_{1}
$$

where

$$
\bar{A}_{i}(t):=W_{i}^{-1} A_{i}(t) W_{i}
$$

We conclude that

$$
\left|z_{1}(t)\right| \leq \widetilde{\alpha}_{i} e^{-\alpha_{i} t}, \quad \widetilde{\alpha}_{i}:=\left|W_{i}^{-1}\right| \bar{\alpha}_{i}, \quad \forall t \geq 0 .
$$

Based on this fact we may now state the following result for the switched error systems which model the network of systems with switching topology.

Lemma 2: Consider the switched system

$$
\dot{z}_{1}=\bar{A}_{\sigma(t)}(t) z_{1}
$$


with $\sigma: \mathbb{R}_{\geq 0} \rightarrow\{1, \ldots, N !\}$ and for each $i \in\{1, \ldots, N !\}$, $\bar{A}_{i}$ is defined in (27). Let the dwell time

$$
\tau_{d}>\frac{\ln \left(\prod_{i=1}^{N !} \widetilde{\alpha}_{i}\right)}{\sum_{i=1}^{N !} \alpha_{i}}
$$

Then, the equilibrium $\left\{z_{1}=0\right\}$ of (26) is uniformly globally exponentially stable for any switching sequence $\left\{t_{p}\right\}$ such that $t_{p+1}-t_{p}>\tau_{d}$ for every switching time $t_{p}$.

Proof: Let $t_{p}$ be an arbitrary switching instant. For all $t \geq t_{p}$ such that $\sigma(t)=i$ we have

$$
\left\|z_{1}(t)\right\| \leq \widetilde{\alpha}_{i} e^{-\alpha_{i}\left(t-t_{p}\right)}\left\|z_{1}\left(t_{p}\right)\right\|, \quad \forall t_{p} \leq t<t_{p+1} .
$$

Since by hypothesis $\tau_{d} \in\left[t_{p}, t_{p+1}\right)$, from (31) we have

$$
\left\|z_{1}\left(t_{p}+\tau_{d}\right)\right\| \leq \widetilde{\alpha}_{i} e^{-\alpha_{i} \tau_{d}}\left\|z_{1}\left(t_{p}\right)\right\| .
$$

Using the property of continuity of both the norm function and the state $z(t)$, we have

$$
\left\|z_{1}\left(t_{p+1}\right)\right\| \leq\left\|z_{1}\left(t_{p}+\tau_{d}\right)\right\|
$$

and therefore

$$
\left\|z_{1}\left(t_{p+1}\right)\right\| \leq \widetilde{\alpha}_{i} e^{-\alpha_{i} \tau_{d}}\left\|z_{1}\left(t_{p}\right)\right\| .
$$

Note that to guarantee asymptotic stability of (29) it is sufficient that for every pair of switching times $t_{p}$ and $t_{q}$

$$
\left\|z_{1}\left(t_{q}\right)\right\|-\left\|z_{1}\left(t_{p}\right)\right\|<0
$$

whenever $p<q$ and $\sigma\left(t_{p}\right)=\sigma\left(t_{q}\right)$.

Now consider the sequence of switching times $t_{p}, t_{p+1}$, $\ldots, t_{p+N !-1}, t_{p+N}$ ! satisfying $\sigma\left(t_{p}\right) \neq \sigma\left(t_{p+1}\right) \neq \ldots \neq$ $\sigma\left(t_{p+N !-1}\right)$ and $\sigma\left(t_{p}\right)=\sigma\left(t_{p+N !}\right)$ which corresponds to a switching signal in which all the $N$ ! switched are chosen.

From (34) it follows that

$$
\left\|z_{1}\left(t_{p+N !}\right)\right\| \leq\left(\prod_{i=1}^{N !} \widetilde{\alpha}_{i} e^{-\left(\sum_{i=1}^{N !} \alpha_{i}\right) \tau_{d}}\right)\left\|z_{1}\left(t_{p}\right)\right\| .
$$

To ensure that

$$
\left\|z_{1}\left(t_{p+N !}\right)\right\|-\left\|z_{1}\left(t_{p}\right)\right\|<0
$$

it is sufficient that

$$
\left(\prod_{i=1}^{N !} \widetilde{\alpha}_{i} e^{-\left(\sum_{i=1}^{N !} \alpha_{i}\right) \tau_{d}}-1\right)<0
$$

Therefore, since the norm is a non-negative function we obtain

$$
\prod_{i=1}^{N !} \widetilde{\alpha}_{i} e^{-\left(\sum_{i=1}^{N !} \alpha_{i}\right) \tau_{d}}<1
$$

and the proof follows.

Finally, in view of Lemma 2 we can make the following statement.

Theorem 1: Let $\left\{t_{p}\right\}$ denote a sequence of switching instants $p \in \mathbb{Z}_{\geq 0}$ and let $\sigma: \mathbb{R}_{\geq 0} \rightarrow\{1, \ldots, N$ ! $\}$ be a piecewise constant function satisfying $\sigma(t) \equiv i$ for all $t \in$ $\left[t_{p}, t_{p+1}\right)$ with $t_{p}-t_{p+1} \geq \tau_{d}$ and $\tau_{d}$ satisfying (30).
Consider the system (1) in closed loop with

$u_{\pi_{k}}^{\sigma(t)}=\left\{\begin{array}{cl}-a_{\pi_{k} \pi_{k+1}}^{\sigma(t)}(t)\left[x_{\pi_{k}}-x_{\pi_{k+1}}\right], & k \in\{1, \ldots, N-1\}, \\ 0, & k=N .\end{array}\right.$

Let the interconnection gains $a_{\lambda \kappa}^{i}$, for all $i \in\{1, \ldots, N !\}$ and all $\lambda, \kappa \in\{1, \ldots, N-1\}$, be persistently exciting. Then, the system reaches consensus with uniform exponential convergence.

\section{EXAMPLE}

For illustration, we consider a network of three agents hence, with six possible topologies, as showed in Figure 1. The information exchange among agents in each topology is ensured via channels with persistently-exciting communication intensity; the corresponding parameters are shown in Table I.

\begin{tabular}{|l|c|c||l|c|c||l|c|c|}
\hline $\mathrm{i}=1$ & $T$ & $\mu$ & $\mathrm{i}=2$ & $T$ & $\mu$ & $\mathrm{i}=3$ & $T$ & $\mu$ \\
\hline$a_{12}(t)$ & 0.25 & 0.5 & $a_{21}(t)$ & 2.0 & 1.6 & $a_{31}(t)$ & 0.3 & 0.1 \\
\hline$a_{23}(t)$ & 0.2 & 1 & $a_{13}(t)$ & 0.8 & 0.2 & $a_{12}(t)$ & 0.7 & 0.6 \\
\hline \hline $\mathrm{i}=4$ & $T$ & $\mu$ & $\mathrm{i}=5$ & $T$ & $\mu$ & $\mathrm{i}=6$ & $T$ & $\mu$ \\
\hline$a_{13}(t)$ & 2 & 1 & $a_{23}(t)$ & 0.4 & 0.1 & $a_{32}(t)$ & 0.5 & 0.3 \\
\hline$a_{32}(t)$ & 4 & 0.4 & $a_{31}(t)$ & 0.5 & 0.4 & $a_{21}(t)$ & 4.2 & 1.8 \\
\hline
\end{tabular}

Table I. Parameters of the interconnection gains.

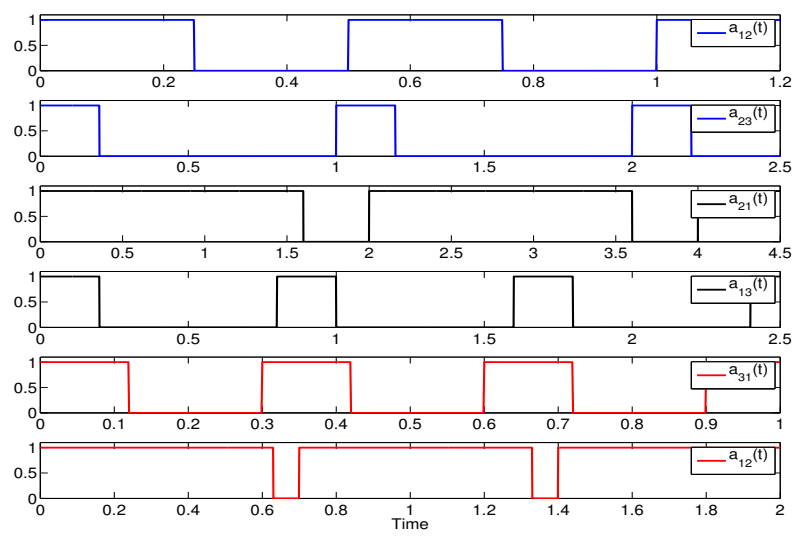

Fig. 6. Persistently exciting interconnection gains for the topologies $\left\{\Psi_{1}, \Psi_{2}, \Psi_{3}\right\},\left\{\Psi_{2}, \Psi_{1}, \Psi_{3}\right\}$ and $\left\{\Psi_{3}, \Psi_{1}, \Psi_{2}\right\}$.

The graphs corresponding to the interconnection gains are showed in Figures 6 and 7. By applying Lemma 1, we can compute $\widetilde{\alpha}_{i}$ and $\alpha_{i}$ for each topology $i$, see Table II. Substituting the values of $\widetilde{\alpha}_{i}$ and $\alpha_{i}$ into (30), we find that the dwell time must satisfy $\tau_{d}>7.92$.

\begin{tabular}{|c|c|c|c|c|c|c|}
\hline \hline $\mathrm{i}$ & 1 & 2 & 3 & 4 & 5 & 6 \\
\hline$\widetilde{\alpha}_{i}$ & 6.51 & 10.26 & 6.11 & 12.85 & 4.71 & 3.98 \\
$\alpha$ & 0.2 & 0.3 & 0.4 & 0.1 & 0.35 & 0.1 \\
\hline
\end{tabular}

Table II. Parameters corresponding to the exponential bounds.

We performed some numerical simulations using Simulink of MATLAB. In a first test, the initial conditions are set to $x_{1}(0)=-2, x_{2}(0)=1.5$ and $x_{3}(0)=-0.5$; the switching signal $\sigma(t)$ is illustrated in Figure 8. The systems' trajectories, converging to a consensus equilibrium, are showed in Figure 9. It is worth mentioning, however, that the dwell-time condition (30) only provides a sufficient stability condition. In Figure 10 we show the 
graph of a switching signal which does not respect the dwell-time condition and, yet, all the states converge to a common value -see Figure 11.

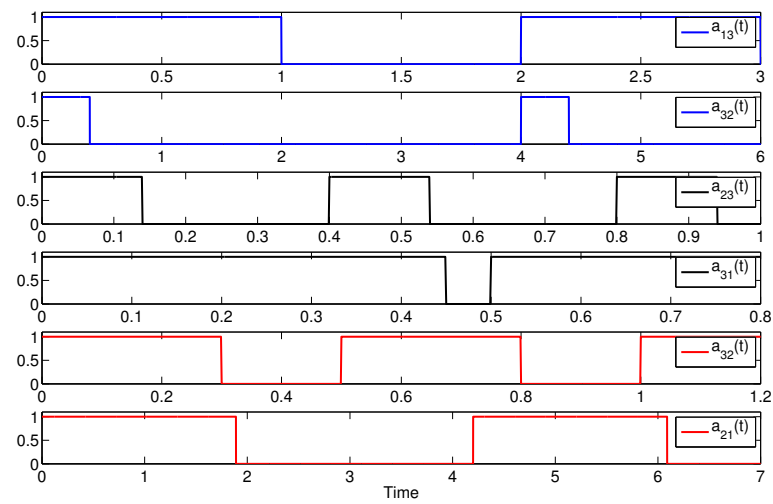

Fig. 7. Persistently exciting interconnection gains for the topologies $\left\{\Psi_{1}, \Psi_{3}, \Psi_{2}\right\},\left\{\Psi_{2}, \Psi_{3}, \Psi_{1}\right\}$ and $\left\{\Psi_{3}, \Psi_{2}, \Psi_{1}\right\}$.

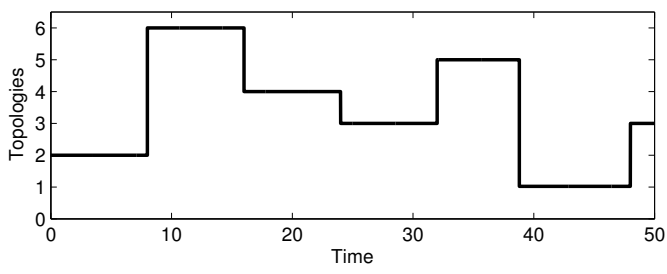

Fig. 8. A switching signal $\sigma(t)$ satisfying the dwell-time condition.

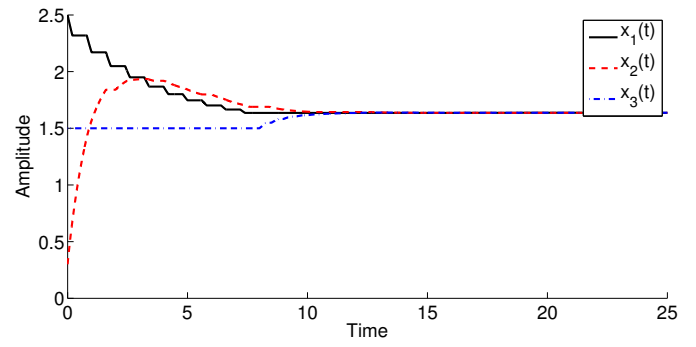

Fig. 9. Trajectories of $x_{1}, x_{2}$ and $x_{3}$.

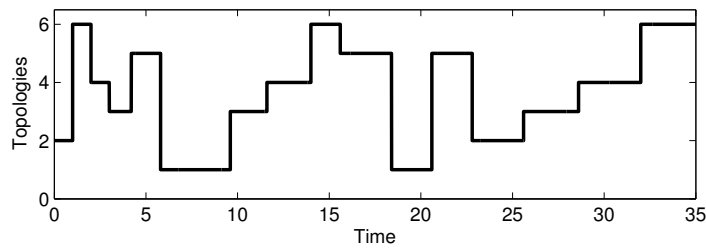

Fig. 10. The switching signal $\sigma(t)$, which does not satisfy the dwell-time condition.

\section{CONCLUSIONS}

We provided the convergence analysis of a consensus problem for a network of integrators with directed information flow under time-varying topology. Our analysis relies on stability theory of time-varying and switched systems. We established a minimal dwell-time conditions over the switching signal.

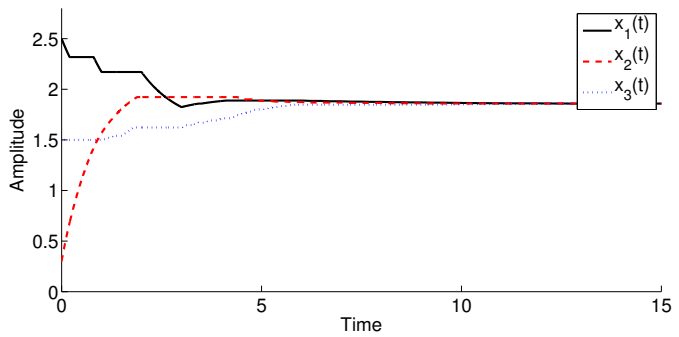

Fig. 11. Trajectories of $x_{1}, x_{2}$ and $x_{3}$.

\section{REFERENCES}

[1] J.R. Carpenter. Decentralized control of satellite formations. Int. J. Robust Nonlinear Control, 12(2):141-161, 2002.

[2] N. R. Chowdhury, S. Sukumar, and N. Balachandran. Persistence based convergence rate analysis of consensus protocols for dynamic graph networks. European Journal of Control, 29:33-43, 2016.

[3] N. R. Chowdhury, S. Sukumar, M. Maghenem, and A. Lloría. On the estimation of algebraic connectivity in graphs with persistently exciting interconnections. Int. J. of Contr., 91(1):131-144, 2018. Pre-published online Jan 2017. DOI: 10.1080/00207179.2016.1272006.

[4] J. Dasdemir and A. Lorìa. A simple formation-tracking controller of mobile robots based on a "spanning-tree" communication. 51st IEEE Conf. Decision and Control, pages $1796-1801,2012$.

[5] Y. Gong, L. Wang, and G. Xie. Average consensus in networks of dynamic agents with switching topologies and multiple time-varying delays. Systems and Control Letters, 57(2):175$183,2007$.

[6] R. A. Horn and C. R. Johnson. Matrix Analysis. Cambridge University Press, 1985.

[7] HongKeun Kim, Hyungbo Shim, Juhoon Back, and Jin Heon Seo. Consensus of output-coupled linear multi-agent systems under fast switching network: Averaging approach. Automatica, 49(1):267-272, 2013.

[8] S. A. Kumar, N. R. Chowdhury, S. Srikant, and J.Raisch. Consensus analysis of systems with time-varying interactions : An event-triggered approach. IFAC-PapersOnLine, 50(1):93499354, 2017.

[9] M. Maghenem and A. Loría. Lyapunov functions for persistently-excited cascaded time-varying systems: application in consensus analysis. IEEE Trans. on Automatic Control, 62(7):3416-3422, 2017. DOI: 10.1109/TAC.2016.2610099.

[10] L. Moreau. Stability of continuous-time distributed consensus algorithms. 43rd IEEE Conf. Decision and Control, 4:3998 $-4003,2004$.

[11] R. Olfati-Saber and R. M. Murray. Consensus problems in networks of agents with switching topology and time-delays. IEEE Trans. on Automatic Control, 49:1520-1533, 2004.

[12] W. Ren and R. W. Beard. Consensus seeking in multi-agent systems under dynamically changing interaction topologies. IEEE Trans. on Automatic Control, 50(5):655-661, 2005.

[13] W. Ren and R. W. beard. Distributed consensus in multivehicle cooperative control. Springer verlag, 2005.

[14] A. Sarlette, R. Sepulchre, and N.E. Leonard. Cooperative attitude synchronization in satellite swarms: a consensus approach. in Proc. 17th IFAC Symp. Autom. Control Aerosp., pages 223-228, 2007.

[15] C. Tomlin, G.J. Pappas, and S. Sastry. Conflict resolution for air traffic management: A study in multiagent hybrid systems communication. IEEE Trans. Autom. Contr., 43(4):509-521, 1998.

[16] F. Xiao and L. Wang. State consensus for multi-agent systems with switching topologies and time-variant delays. International Journal of Control, 79(10):1277-1284, 2006.

[17] Keyou You, Zhongkui Li, and Lihua Xie. Consensus condition for linear multi-agent systems over randomly switching topologies. Automatica, 49(10):3125-3132, 2013. 